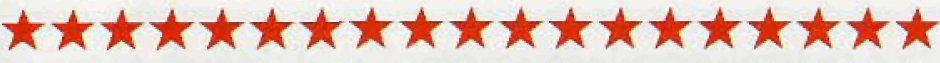
back to our met-lab several times before a satisfactory surface is available. A very light "attack" polish (not really an etch) can be helpful during final polish for some materials. I have wanted to try a light ion beam cleaning, but don't have one.

5. Incident beam angle (with specimen at $90^{\circ}$ nominal) changes resulting from the raster can strongly affect contrast. Experiment with working distance vs. magnification to see what works best for a given specimen.

Along this line, don't expect to be able to generate multiple image mosaics which match well. A grain that is dark on one edge of an image may will be light when the stage is translated one frame over because the incident angle is no longer the same.

N.W. White, Jr. (Woody), Babcock \& Wilcox Co.

\section{Aligning Ion Guns}

This method requires a long-working distance binocular microscope on the top of the ion gun, that allows you to observe your sample as it thins, and an electromagnetic gun alignment system that allows you to align the ion beams from outside the vacuum. However, it requires minimal handling and no chemicals or other special equipment.

Guns are generally aligned after cleaning and anode replacement.

1. In place of the sample and tantalum sample-holding plates, place a glass cover slip $10 \mathrm{~mm}$ in diameter and screw down the holder as usual. A small cross in the centre of the glass slide is helpful. Place the holder in the stage, insert the stage in the ion mill and pump down. Rotate the stage at low speed.

2. Use a gun tilt of about 22 degrees, turn on just the ' $A$ ' gun to $6 \mathrm{kV}$ and 40 nA current. You should see the glass coverslip fluoresce where the beam hits it. Align, using the ' $X$ ' and ' $Y$ ' knobs for gun ' $A$ ' on the alignment control box to centre the beam on the rotation centre of the glass slip.

3. Turn off gun ' $A$ ' and turn on gun ' $B$ ' to the same conditions. Align using the control box controls for the 'B' gun.

Mary Mager, University of British Columbia

(This is the method advised by VCR to align the guns of their lon Mill)

Note: Paul Fischione, E.A. Fischione Instruments, Inc. recommends using the thinnest coverslips available, as alignment is done using the fluorescence from both top and bottom surfaces of the coverslip. Thicker slips can lead to centering errors because of this.

\section{Using Liquid $\mathbf{N}_{2}$ To "Pop" Resin-embedded Cell Cultures Grown On Coverslips}

We have "popped" cells grown in both serum and serum-free media on glass coverslips using liquid nitrogen hundreds of times. Serum makes no difference. The coverslips are fixed either in the 12-well plastic trays or transferred to $20 \mathrm{~mL}$ glass scintillation vials. It you leave the coverslips in the plastic trays during infiltration, you run the risk of extracting something that interferes with the polymerization. To avoid this problem, we always switch to glass vials by the time we start dehydration.

After $100 \%$ ethanol:

infiltrate with Embed 812 (1:1 with ethanol overnight)

then $100 \%$ resin for 3 hours

then place the coversllip cell side up on a glass slide with the thin layer of resin that comes with it when transferred to the slide. Not too thin or too thick but, if in doubt, simply add 1 drop of resin to the coverslip after transferring and let it flow naturally. Final plastic spread should be no more than 1.5 - 2 times the diameter of the coverslip.

When the slides are taken out of the oven, let them cool for 15 minutes, and touch with a razor blade. If a gooey strand comes with the blade when it is pulled away, it hasn't $t$ been polymerized enough.

It it is not too gooey, cross-hatch the surface of the plastic using the razor blade to score deeply into the plastic (to the level of the glass).
Slowly immerse the slide into liquid $\mathrm{N}_{2}$ and the squares should pop right off.

Look at the surface the coverslip and bottom of the square to ensure no glass is going with the square. It so, it has been over polymerized.

For some projects, we simply put the square in the flat microtome holder and cut it, but usually we re-embed the square in a standard mold. If the specimens aren't to be re-embedded, heat the squares in a rubber mold overnight. Once the timing of how long to polymerize the coverslips is found, it is trivial. We haven't had a failure in years of doing this

Thomas E. Phillips, University of Missouri

\section{A Caveat About SEM Sample Storage Boxes}

While the highly popular storage boxes made with the soft elastomeric base plate (such as "SPI boxes") are attractive for most SEM users, the plasticizer found In the elastomeric plastic can cause problems with storing some samples if they are reactive (such as those prepared using the Osmium-Thiocarbazide-Osmium method).

The soft elastomeric ones (e.g., those made by SPI) are made that way specifically so that the holes "stretch" sort of elastically, like a rubber band, so the holes don't lose their grip. Therefore, the useful lifetime of these boxes is almost forever. However, the base plate into which the mounts are placed also contains a plasticizer, and In the presence of the $\mathrm{OSO}_{4}$ vapors (apparently) some kind of reaction occurs, resulting in a fine, somewhat feathery deposition onto the mounted and prepared samples. I have never found anyone who has been able to resurrect such samples to the degree that they could ever be examined again

The boxes with the hard, rigid plastic base plates are much more inert and will cause much less of a problem when used to store O-T-O treated samples. Samples being so stored might not last forever, but they should survive a very long time.

One might ask, why make boxes with soft elastomeric base plates, why not only boxes with hard, rigid plastic base plates? Anyone who has used them will know that after putting in and removing mounts more than a few times, the holes tend to develop a permanent set, and then mounts don't stay put when the box is turned upside-down.

Charles A. Garber, SPI Supplies/Structure Probe, Inc.

\section{Flat Embedding Specimens In Media Which Polymerize under UV}

We have used both LR White and Lowicryl resins successfully for flat embedding. We use Been flat embedding molds, which are quite stiff and seem to contain less free oxygen than standard molds.

Our trick to exclude atmospheric oxygen is to use the plastic pipette tip box that "Multi-Flex" tips are packaged in. It is a three part box consisting of a UV light penetrable top, which fits over a multi-holed mid section (normally supporting the tips) which fits over a lower compartment. (I collected a bunch of these from another lab, but I do not know where to get them.)

The lower compartment is filled with dry ice (solid $\mathrm{CO}_{2}$ ), and drill a couple of holes in the top (letting $\mathrm{CO}_{2}$ gas escape as the solid sublimes). The samples in the beem flat embedding molds are placed on the perforated "shelf" above the $\mathrm{CO}_{2}$ chamber and exposed to a constantly replenished environment of gaseous $\mathrm{CO}_{2}$, excluding incoming oxygen.

The whole thing, including a UV light, Is put In the $-20^{\circ} \mathrm{C}$ portion of the freezer. The polymerization proceeds fully enough by the time the dry ice is gone to prevent oxygen inhibition. You might also try using a "heat sink" type of mold

Doug Keene, Shriners Hospital EM Lab 